\title{
MaioRegen Osteochondral Substitute for the Treatment of Knee Defects: A Systematic Review of the Literature
}

\author{
Riccardo D'Ambrosi ${ }^{1}{ }^{1 * \mathbb{D}}$, Federico Valli ${ }^{1}$, Paola De Luca ${ }^{2}$, Nicola Ursino ${ }^{1}$ and \\ Federico Giuseppe Usuelli ${ }^{1}$ (D) \\ 1 IRCCS Istituto Ortopedico Galeazzi, CASCO Department, 20161 Milan, Italy; mail@federicovalli.it (F.V.); \\ nicolaursino@libero.it (N.U.); fusuelli@gmail.com (F.G.U.) \\ 2 IRCCS Istituto Ortopedico Galeazzi, Orthopaedic Biotechnology Lab, 20161 Milan, Italy; \\ deluca.paola@grupposandonato.it \\ * Correspondence: riccardo.dambrosi@hotmail.it
}

Received: 1 May 2019; Accepted: 28 May 2019; Published: 1 June 2019

\begin{abstract}
Background: This study aims to investigate the clinical and radiological efficacy of three-dimensional acellular scaffolds (MaioRegen) in restoring osteochondral knee defects. Methods: MEDLINE, Scopus, CINAHL, Embase, and Cochrane Databases were searched for articles in which patients were treated with MaioRegen for osteochondral knee defects. Results: A total of 471 patients were included in the study (mean age $34.07 \pm 5.28$ years). The treatment involved 500 lesions divided as follows: 202 (40.4\%) medial femoral condyles, 107 (21.4\%) lateral femoral condyles, $28(5.6 \%)$ tibial plateaus, $46(9.2 \%)$ trochleas, $74(14.8 \%)$ patellas, and $43(8.6 \%)$ unspecified femoral condyles. Mean lesion size was $3.6 \pm 0.85 \mathrm{~cm}^{2}$. Only four studies reported a follow-up longer than 24 months. Significant clinical improvement has been reported in almost all studies with further improvement up to 5 years after surgery. A total of 59 complications were reported of which $52(11.1 \%)$ experienced minor complications and 7 (1.48\%) major complications. A total of 16 (3.39\%) failures were reported. Conclusion: This systematic review describes the current available evidence for the treatment of osteochondral knee defects with MaioRegen Osteochondral substitute reporting promising satisfactory and reliable results at mid-term follow-up. A low rate of complications and failure was reported, confirming the safety of this scaffold. Considering the low level of evidence of the study included in the review, this data does not support the superiority of the Maioregen in terms of clinical improvement at follow-up compared to conservative treatment or other cartilage techniques.
\end{abstract}

Keywords: osteochondral defect; scaffold; cartilage; graft; MaioRegen; osteochondral substitute

\section{Introduction}

Achieving a predictable and durable repair after an articular cartilage knee injury is still a clinical challenge for orthopedic surgeons. In recent years, advancements in arthroscopy and imaging have led to an increase in the acute recognition of chondral and osteochondral defects [1-3]. Curl et al. reported a percentage of $63 \%$ of patients with chondral injury considering 31,516 knee arthroscopies; this condition affects approximately 900,000 Americans annually resulting in more than 200,000 surgical procedures [4,5]. Cartilage injuries can result in pain, swelling, clicking, instability, and finally progression to a more diffuse degenerative process [6,7]. Current surgical treatment includes arthroscopic debridement and arthroscopic bone marrow stimulation including microfractures, used either alone or in combination with scaffold such as in the case of the autologous matrix-induced chondrogenesis (AMIC) technique that combines microfractures with a collagen I/III membrane [8]. Other treatments include autologous or allogenic osteochondral transplantation as well as natural 
or synthetic scaffolds used alone or in combination with cells. Finally, cell-based therapies, such as autologous chondrocyte implantation (ACI) represent an effective, although expensive clinical treatment for patients affected by focal chondral lesions. More recently, second-generation methods to improve the outcome of the ACI procedure using three-dimensional scaffolds have been described (MACI, matrix-induced ACI) [9-11].

Although the use of allogenic osteochondral grafts might appear a good strategy, the complex biological events behind allograft integration as well as the lack of availability of allografts in many countries limit their use [12]. To overcome these drawbacks, the last few years have seen the development of new bioengineered scaffolds for the treatment of cartilage defects [10]. When implanting an osteochondral scaffold at the lesion site, the aim is to promote tissue repair through the formation of a tissue that is as similar as possible to the native one, with physiological properties similar to those of the entire osteochondral unit, and therefore durable over time [11]. In recent years new biomimetic three-dimensional acellular scaffolds (MaioRegen; Fin-Ceramica Faenza SpA) have been proposed for "in situ" cartilage regeneration [13]. MaioRegen is a nanostructured biomimetic and bioresorbable implant with a porous composite structure, mimicking the whole osteochondral anatomy with its three different layers:

- $\quad$ superficial layer (100\% type I collagen): smooth surface, reproducing the articular surface.

- intermediate layer (60\% type I collagen and $40 \%$ hydroxyapatite): tidemark-like layer.

- lower layer (30\% type I collagen and $70 \%$ hydroxyapatite): reproducing the composition of the subchondral bone [13].

Currently in the literature, there is still no evidence regarding the use of MaioRegen in the treatment of osteochondral knee defects, despite numerous articles reporting its use; in particular, the literature is scarce regarding the randomized clinical trials.

This systematic review aims to investigate the clinical and radiological efficacy of MaioRegen in restoring osteochondral knee defects.

\section{Experimental Section}

\subsection{Data Search Protocol}

A systematic review of the existing literature was performed to identify all studies dealing with MaioRegen scaffold for the treatment of chondral and osteochondral knee defects. The Preferred Reporting Items for Systematic Reviews and Meta-analyses (PRISMA) guidelines were followed for the identification of the articles [14]. The research was performed by two independent investigators using MEDLINE, Scopus, CINAHL, Embase, and Cochrane Databases up to June 2018. Main search items were "MaioRegen", "biomemetic scaffold", "multilayered scaffold", "scaffold", "osteochondral lesion", "cell-free scaffold", "biocomposite scaffold". The complete search strategy is shown in Figure 1. Additionally, reference lists of the selected articles were screened for further publications.

\subsection{Study Selection and Eligibility Criteria}

This systematic review includes studies of level I to IV (according to the "The Oxford 2011 Levels of Evidence" [15]).

The articles were analyzed regardless of their title and abstract by the two investigators. In case of disagreement, the papers were discussed until agreement was reached. In this systematic review, the inclusion criteria were: article that reported clinical, histological or radiological outcome data in patients treated with MaioRegen scaffold for osteochondral knee defect, article written in English, study of level I, II, III or IV; article published between January 1990 and June 2018.

Exclusion criteria were: article not written in English, case series with less than 5 cases, case reports, editorials, systematic reviews, and meta-analysis. 


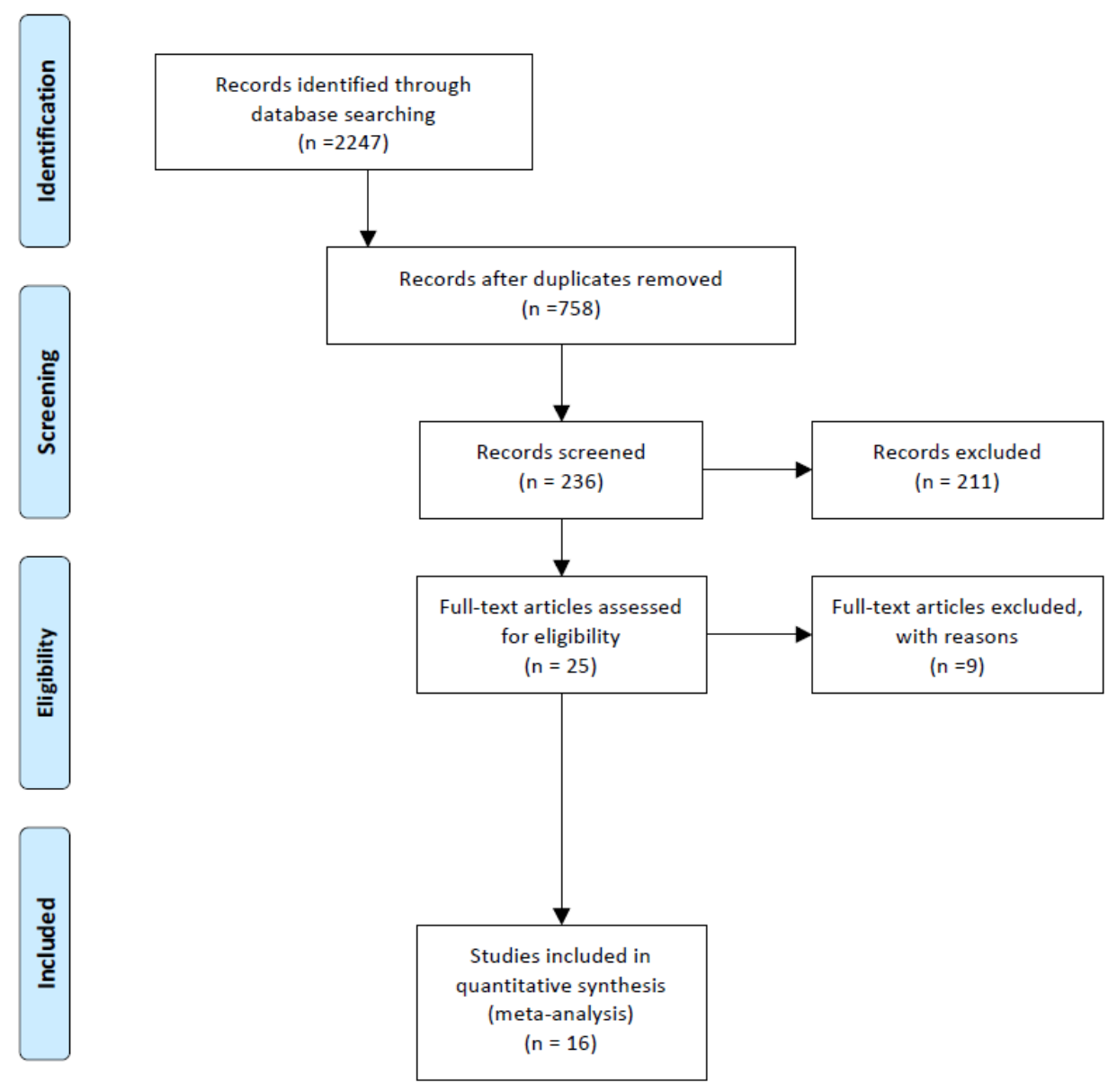

Figure 1. A flowchart of the literature screening performed in this study.

\subsection{Data Extraction, Synthesis, and Analysis}

Three independent reviewers analyzed all the information available from the articles (data and journal of publication, type of the study level of evidence, demographics data, diagnosis, surgical procedure, follow-up duration, outcomes, and complications) and entered it into a spreadsheet for analysis. Inter-observer agreement, was 0.95 (PRISMA guidelines) [16].

Results from the early postoperative period (12 months), intermediate postoperative period (12-24 months), and long-term follow-up results ( $>24$ months) were gathered. Radiological information about defect filling, integration of newly formed cartilage with the adjacent cartilage, the cartilage surface quality, and the properties of the subchondral bone was extracted. Moreover, the rate of failure meant as the need for successive knee arthroplasty or realignment surgery was recorded. Complications, such as adverse reactions, infections, or patients who underwent a second surgery (including knee mobilization) were collected too. Concomitant surgeries were not considered in the study.

\section{Results}

The initial search resulted in 2,247 articles, of which 16 articles were selected based on the eligibility criteria (Figure 1). Patient characteristics of the included studies are shown in Table 1 [17-32]. Of the 16 studies, 15 [17-21,23-32] (93.75\%) were case series (Level IV) and only one [22] (6.25\%) a comparative study (Level III). All articles were published between 2011 and 2018. Mean number of patients for the study was $29.44 \pm 17.66$. 
Table 1. Clinical studies investigating the use of MaioRegen for osteochondral knee defects.

\begin{tabular}{|c|c|c|c|c|c|c|c|c|c|}
\hline \multirow[t]{2}{*}{ Article } & \multirow[t]{2}{*}{ Type of Study } & \multirow{2}{*}{$\begin{array}{l}\text { Number of } \\
\text { Patients/ } \\
\text { Mean Age }\end{array}$} & \multirow[t]{2}{*}{ Location } & \multirow[t]{2}{*}{ Lesion Size } & \multirow[t]{2}{*}{ Follow-Up } & \multicolumn{2}{|c|}{ Clinical Results } & \multirow{2}{*}{$\begin{array}{c}\begin{array}{c}\text { Radiologic Results/ } \\
\text { Histological } \\
\text { Evaluation }\end{array} \\
\text { Postop }\end{array}$} & \multirow[t]{2}{*}{ Complications/Reoperation } \\
\hline & & & & & & Preop & Postop & & \\
\hline $\begin{array}{l}\text { Delcogliano et al. } \\
\text { 2014, KSSTA [17] }\end{array}$ & $\begin{array}{l}\text { Case Series, } \\
\text { Level IV }\end{array}$ & $\begin{array}{c}19 \text { patients; } 33 \\
\pm 15 \text { years }\end{array}$ & $\begin{array}{l}20 \text { lesions: } \\
\text { 10 MFC, } \\
7 \text { LFC, } \\
3 \text { tibial plateau }\end{array}$ & $\begin{array}{l}5.2 \pm 1.6 \mathrm{~cm}^{2} . \\
\left(4-8 \mathrm{~cm}^{2}\right) ; \text { mean } \\
\text { depth of } 8 \mathrm{~mm} \\
(6-9 \mathrm{~mm})\end{array}$ & $\begin{array}{l}12 \text { and } 24 \\
\text { months }\end{array}$ & $\begin{array}{l}\text { IKDC: } 35.7 \pm 6.3 \\
\text { Tegner: } 2(0-4) \\
\text { EQ-VAS: } 3.1 \pm 1.1\end{array}$ & $\begin{array}{c}\text { IKDC: } \\
12 \text { months: } 67.7 \pm 13.4(p<0.0005)^{*} * \\
24 \text { months: } 72.8 \pm 12.4(p<0.0005)^{*} \\
\text { Tegner: } \\
24 \text { months: } 5(1-7)(p<0.0005)^{*} \\
\text { EQ-VAS } \\
24 \text { months: } 7.3 \pm 1.1(p<0.0005)^{*}\end{array}$ & $\begin{array}{c}\text { MOCART } \\
24 \text { months: } 63.2 \pm 11.7\end{array}$ & $\begin{array}{c}2 \text { failures: } \\
12 \text { and } 24 \text { months after surgery } \\
\text { No complications }\end{array}$ \\
\hline $\begin{array}{l}\text { Brix et al. 2016, Int } \\
\text { Orthop [18] }\end{array}$ & $\begin{array}{l}\text { Case Series, } \\
\text { Level IV }\end{array}$ & $\begin{array}{c}8 \text { patients; } 37 \\
\text { years }\end{array}$ & $\begin{array}{l}8 \text { lesions: } \\
5 \text { MFC, } \\
3 \text { LFC }\end{array}$ & $\begin{array}{c}2.07 \mathrm{~cm}^{2}(1.5 \text { to } \\
\left.3.75 \mathrm{~cm}^{2}\right)\end{array}$ & $\begin{array}{l}12,18 \text { and } 24 \\
\text { months }\end{array}$ & $\begin{array}{c}\text { IKDC } \\
\text { Lysholm } \\
\text { Cincinnati }\end{array}$ & $\begin{array}{c}\text { IKDC: } \\
\text { 6,12,18 and } 24 \text { months: } p=0.30 \\
\text { Tegner-Lysholm: } \\
\text { 6,12,18 and } 24 \text { months: } p=0.176 \\
\text { Cincinnati } \\
\text { 6,12,18 and } 24 \text { months: } p=0.53\end{array}$ & $\begin{array}{c}\text { MOCART } \\
18 \text { months: } 69 \text { (60-100) } \\
\text { T2 mapping at } 18 \\
\text { months: } 0.9874\end{array}$ & $\begin{array}{l}2 \text { complications: resurgery at } 7 \\
\text { and } 24 \text { months not for scaffold } \\
\text { failure. } \\
\text { In the first case the } \\
\text { re-arthroscopy showed a novel } \\
\text { chondral lesion on the lateral } \\
\text { condyle. In the second case an } \\
\text { infrapatellar ossicle was } \\
\text { removed } \\
\text { No failures }\end{array}$ \\
\hline $\begin{array}{l}\text { Berruto et al. 2016, } \\
\text { The Knee [19] }\end{array}$ & $\begin{array}{l}\text { Case Series, } \\
\text { Level IV }\end{array}$ & $\begin{array}{c}11 \text { patients; } \\
52.1 \pm 9.6 \text { years }\end{array}$ & $\begin{array}{l}11 \text { lesions: } \\
9 \text { MFC, } \\
2 \text { LFC }\end{array}$ & $\begin{array}{c}3.47 \pm 1.75 \mathrm{~cm}^{2} \\
\text { (range } 1.5 \text { to } 7.5 \\
\mathrm{~cm}^{2} \text { ) }\end{array}$ & $\begin{array}{c}12 \text { and } 24 \\
\text { months }\end{array}$ & $\begin{array}{c}\text { IKDC: } 40.54 \pm 15.0 \\
(17-57) \\
\text { Lysholm: } 49.7 \pm 17.9 \\
(22-88) \\
\text { Tegner: } 4 \pm 1.1 \\
\text { (2-6)(pre-injury) } \\
\text { VAS: } 6.3 \pm 2.5 \\
(3-8)\end{array}$ & $\begin{array}{c}\text { IKDC: } \\
12 \text { months: } 65.72 \pm 14.8^{*}(p=0.014) \\
24 \text { months: } 63.90 \pm 19.9^{*}(p=0.03) \\
\text { Lysholm: } \\
12 \text { months: } \\
24 \text { months: } 86.4 \pm 12.11^{*}(p=0.01) \\
\text { Tegner: } \\
12 \text { months: } 3.7 \pm 0.04) \\
24 \text { months: } 3.8 \pm 1.7(p>0.05) \\
\text { VAS: }(p>0.05) \\
12 \text { months: } 2.2 \pm 2.1^{*}(p=0.02) \\
24 \text { months: } 1.6 \pm 2.7^{*}(p<0.05)\end{array}$ & N.A. & $\begin{array}{l}2 \text { failures: } 2 \text { condylar collapse } \\
\text { and subsequent knee } \\
\text { arthroplasty at } 18 \text { months after } \\
\text { implantation }\end{array}$ \\
\hline $\begin{array}{l}\text { Kon et al. 2014, } \\
\text { Injury [20] }\end{array}$ & $\begin{array}{l}\text { Case Series, } \\
\text { Level IV }\end{array}$ & $\begin{array}{l}11 \text { patients; } \\
37.3 \pm 11.0 \\
\quad \text { years }\end{array}$ & $\begin{array}{l}13 \text { lesions: } \\
11 \text { tibial plateau, } \\
1 \text { MFC, } \\
1 \text { LFC }\end{array}$ & $\begin{array}{l}5.1 \pm 2.7 \mathrm{~cm}^{2} \\
\left(3.0-12.5 \mathrm{~cm}^{2}\right)\end{array}$ & $\begin{array}{l}6,12 \text { and } 24 \\
\text { months }\end{array}$ & $\begin{array}{l}\text { IKDC: } 42.5 \pm 10.2 \\
\text { Tegner: } 2.3 \pm 2.1\end{array}$ & $\begin{array}{c}\text { IKDC: } \\
6 \text { months: } 58.3 \pm 14.1 *(p<0.05) \\
12 \text { monthss: } 69.8 \pm 190^{* *}(p=0.03) \\
24 \text { months: } 68.4 \pm 17.0^{*} \\
\text { Tegner: } \\
12 \text { months } 4.8 \pm 2.4^{*}(p<0.05) \\
24 \text { months: } 5.3 \pm 2.5^{*}\end{array}$ & N.A. & $\begin{array}{l}3 \text { minor complications: fever } \\
\text { during the first week } \\
\text { spontaneously resolved. }\end{array}$ \\
\hline $\begin{array}{l}\text { Di Martino et al. } \\
\text { 2015, Injury [21] }\end{array}$ & $\begin{array}{l}\text { Case Series, } \\
\text { Level IV }\end{array}$ & $\begin{array}{l}23 \text { patients; } \\
38.0 \pm 8.2 \\
\text { years }\end{array}$ & $\begin{array}{c}23 \text { lesions: } 12 \mathrm{MFC}, \\
9 \mathrm{LFC}, \\
1 \text { tibial plateau, } \\
1 \text { patella }\end{array}$ & $3.2 \pm 1.9 \mathrm{~cm}^{2}$ & $\begin{array}{c}12 \text { and } 24 \\
\text { months }\end{array}$ & $\begin{array}{c}\text { IKDC: } 42.8 \pm 13.8 \\
\text { Tegner score: } 3.3 \pm 2.7 \\
\text { (before injury } 6.1 \pm 2.6 \text { ) }\end{array}$ & $\begin{array}{c}\text { IKDC: } \\
12 \text { months: } 74.3 \pm 17.4^{*}(p<0.0005) \\
24 \text { months } 74.9 \pm 20.4^{*} \\
\text { Tegner: } \\
12 \text { months: } 4.6 \pm 2.2^{*}(p<0.0005) \\
24 \text { months: } 4.7 \pm 2.1^{*}\end{array}$ & $\begin{array}{l}\text { Mocart score: } \\
12 \text { monhts: } \\
72.9 \pm 13.6 \\
24 \text { months: } \\
70.8 \pm 13.2\end{array}$ & $\begin{array}{c}2 \text { complications: } \\
2 \text { patients underwent knee } \\
\text { mobilization under narcosis at } \\
2 \text { and } 4 \text { months } \\
2 \text { failures }\end{array}$ \\
\hline
\end{tabular}


Table 1. Cont.

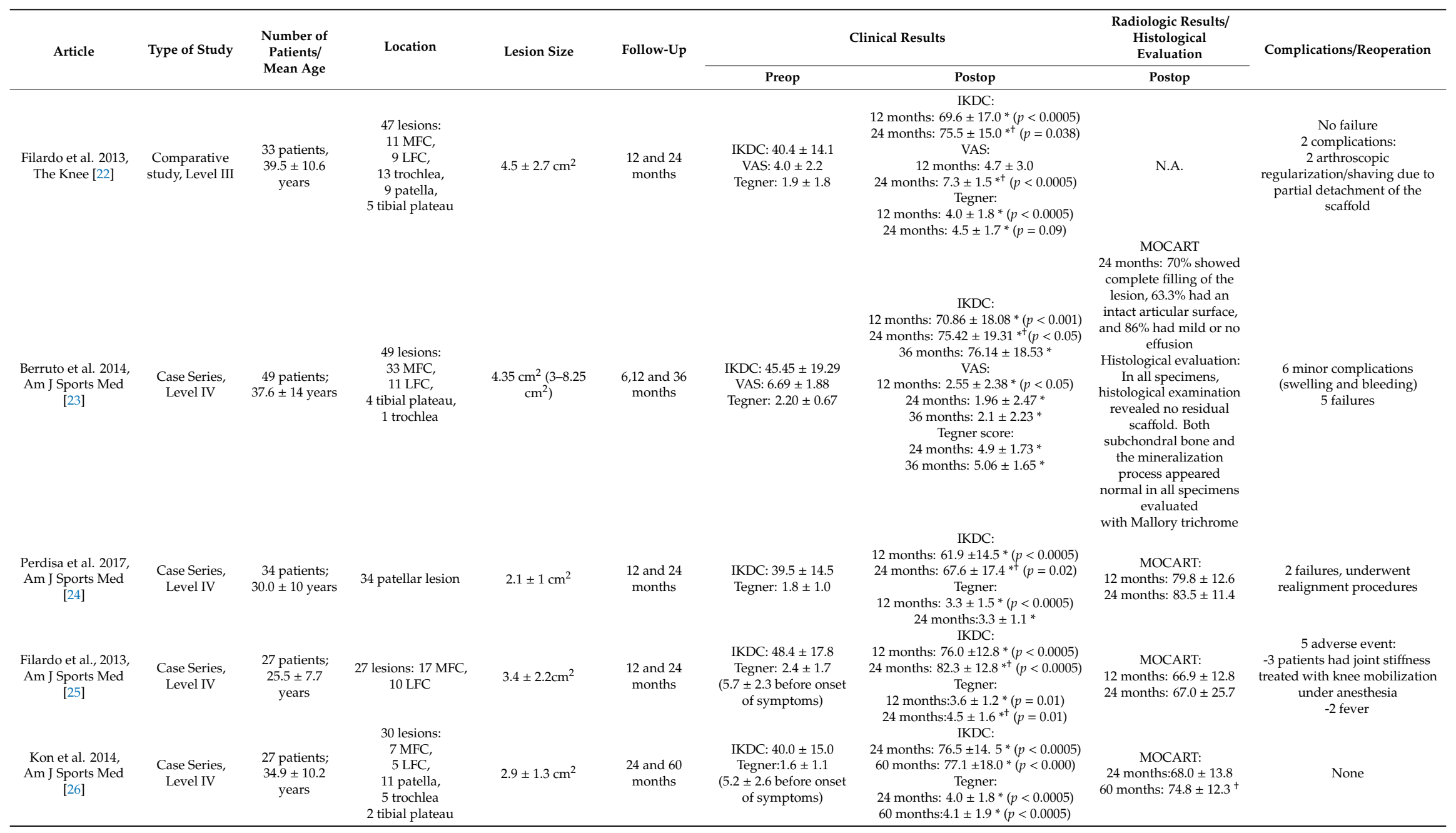


Table 1. Cont

\begin{tabular}{|c|c|c|c|c|c|c|c|c|c|}
\hline \multirow[t]{2}{*}{ Article } & \multirow[t]{2}{*}{ Type of Study } & \multirow{2}{*}{$\begin{array}{l}\text { Number of } \\
\text { Patients/ } \\
\text { Mean Age }\end{array}$} & \multirow[t]{2}{*}{ Location } & \multirow[t]{2}{*}{ Lesion Size } & \multirow[t]{2}{*}{ Follow-Up } & \multicolumn{2}{|c|}{ Clinical Results } & \multirow{2}{*}{$\begin{array}{c}\begin{array}{c}\text { Radiologic Results/ } \\
\text { Histological } \\
\text { Evaluation }\end{array} \\
\text { Postop }\end{array}$} & \multirow[t]{2}{*}{ Complications/Reoperation } \\
\hline & & & & & & Preop & Postop & & \\
\hline $\begin{array}{l}\text { Kon et al. 2011, } \\
\text { Am J Sports Med } \\
\text { [27] }\end{array}$ & $\begin{array}{l}\text { Case Series, } \\
\text { Level IV }\end{array}$ & $\begin{array}{l}28 \text { patients; } \\
35.3 \pm 10.2 \\
\quad \text { years }\end{array}$ & $\begin{array}{l}34 \text { lesions: } \\
8 \mathrm{MFC}, \\
5 \mathrm{LFC}, \\
12 \text { patella, } \\
7 \text { trochlea, } \\
2 \text { tibial plateau }\end{array}$ & $2.9 \pm 1.3 \mathrm{~cm}^{2}$ & $\begin{array}{l}6,12 \text { and } 24 \\
\text { months }\end{array}$ & $\begin{array}{c}\text { IKDC } \\
\text { Tegner: } 1.6 \pm 1.1 \\
(5.2 \pm 2.5 \text { before onset } \\
\text { of symptoms) }\end{array}$ & $\begin{array}{c}\text { IKDC: } \\
\text { 6 months: } p<0.0005^{*} \\
12 \text { months: } p<0.0005^{*} \\
24 \text { months: } p<0.005^{*+} \\
\text { Tegner: } \\
12 \text { months: } 4.0 \pm 1.6^{*}(p<0.0005) \\
24 \text { months: } 4.0 \pm 1.6^{*}(p<0.0005)\end{array}$ & $\begin{array}{c}\text { MOCART: } \\
\text { 24 months: } 79.2 \text { (40-95) } \\
\text { Histological evaluation: } \\
\text { at the e-year follow-up } \\
\text { showed complete } \\
\text { biomaterial } \\
\text { reabsortion and a } \\
\text { hyaline-like tissue with } \\
\text { a strong } \\
\text { proteoglycan content } \\
\text { and presence of collagen } \\
\text { type II }\end{array}$ & $\begin{array}{l}10 \text { complications: Swelling } \\
\text { during the first } \\
\text { month was observed in } 6 \\
\text { patients. One patient } \\
\text { experienced } \\
\text { bleeding during the first } 3 \text { days } \\
\text { after surgery. Two patients } \\
\text { developed a fever during the } \\
\text { first } 3 \text { weeks. All adverse } \\
\text { events resolved within } 1 \\
\text { month after surgery, with the } \\
\text { exception of } 2 \text { patients with } \\
\text { joint stiffness who were } \\
\text { reoperated } \\
\text { on arthroscopically, one at } 2 \\
\text { months and the other at } 5 \\
\text { months. One patient affected } \\
\text { by multiple lesions had } \\
\text { loosening } \\
\text { of one of the grafts, which was } \\
\text { removed, and another } \\
\text { patient was reoperated for } \\
\text { graft hypertrophy. }\end{array}$ \\
\hline $\begin{array}{l}\text { Kon et al. 2014, J } \\
\text { Mater Sci: Mater } \\
\quad \text { Med [28] }\end{array}$ & $\begin{array}{l}\text { Case Series, } \\
\text { level IV }\end{array}$ & $\begin{array}{l}79 \text { patients; } \\
31.0 \pm 11.3 \\
\text { years }\end{array}$ & $\begin{array}{l}82 \text { lesions: } \\
41 \mathrm{MFC}, \\
26 \mathrm{LFC}, \\
15 \text { trochlea }\end{array}$ & $3.2 \pm 2.0 \mathrm{~cm}^{2}$ & $\begin{array}{c}12 \text { and } 24 \\
\text { months }\end{array}$ & $\begin{array}{l}\text { IKDC: } 47.4 \pm 17.1 \\
\text { Tegner: } 2.9 \pm 2.0 \\
\text { (6.3 } 2.2 \text { before the } \\
\text { onset of symptoms) }\end{array}$ & $\begin{array}{c}\text { IKDC: } \\
12 \text { months: } 72.1 \pm 18.9 *(p<0.0005) \\
24 \text { months: } 76.2 \pm 19.6 *+(p=0.004) \\
\text { Tegner: } \\
12 \text { months: } 3.8 \pm 1.6 *(p<0.0005) \\
24 \text { months: } 4.4 \pm 1.9^{* \dagger}(p<0.0005)\end{array}$ & $\begin{array}{c}\text { MOCART: } \\
12 \text { months; median } 70 \\
24 \text { months: median } 80+\end{array}$ & $\begin{array}{l}17 \text { patients reported swelling } \\
9 \text { resurgery due to stiffness }\end{array}$ \\
\hline $\begin{array}{l}\text { Marcacci et al. } \\
\text { 2013, KSSTA [29] }\end{array}$ & $\begin{array}{l}\text { Case Series, } \\
\text { Level IV }\end{array}$ & $\begin{array}{l}43 \text { patients; } \\
40.1 \pm 11\end{array}$ & $\begin{array}{l}43 \text { Femoral } \\
\text { condyles }\end{array}$ & $4.6 \pm 2.1 \mathrm{~cm}^{2}$ & 36 months & $\begin{array}{c}\text { IKDC: } 47.3 \pm 17.1 \\
\text { VAS: } 6.1 \pm 2.0 \\
\text { Tegner: } 2(1-5) \\
\text { 6(3-10 before onset of } \\
\text { symptoms) }\end{array}$ & $\begin{array}{c}\text { IKDC: } 79.6 \pm 16.1^{*}(p<0.0005) \\
\text { VAS: } 2.3 \pm 2.2 *(p<0.0005) \\
\text { Tegner: } 4(3-10)^{*}(p<0.0005)\end{array}$ & N.A. & None \\
\hline $\begin{array}{l}\text { Mathis et al. 2018, } \\
\text { KSSTA [30] }\end{array}$ & $\begin{array}{l}\text { Case Series, } \\
\text { Level IV }\end{array}$ & $\begin{array}{l}14 \text { patients; } 33 \\
\pm 10 \text { years }\end{array}$ & $\begin{array}{l}14 \text { lesions: } \\
8 \text { MFC, } \\
2 \text { CFL. } \\
2 \text { trochlea, } \\
2 \text { patella. }\end{array}$ & $1.0-3.5 \mathrm{~cm}^{2}$ & 12 months & $\begin{array}{l}\text { Lysholm: } 65.6 \pm 12.6 \\
\text { Tegner: } 6.0(3-9)\end{array}$ & $\begin{array}{c}\text { Lysholm: } 90.1 \pm 10.0^{*}(p<0.001) \\
\text { Tegner: } 4.5(p<0.01)^{*}\end{array}$ & $\begin{array}{l}\text { SPECT/CT: A complete } \\
\text { filling of the defect was } \\
\text { shown in } 14 \% \text {, a partial } \\
\text { filling in } 14 \% \text { and only } \\
\text { minor filling } \\
\text { was seen in } 72 \% \text {. }\end{array}$ & None \\
\hline
\end{tabular}


Table 1. Cont.

\begin{tabular}{|c|c|c|c|c|c|c|c|c|c|}
\hline \multirow{2}{*}{ Article } & \multirow[t]{2}{*}{ Type of Study } & \multirow{2}{*}{$\begin{array}{l}\text { Number of } \\
\text { Patients/ } \\
\text { Mean Age }\end{array}$} & \multirow[t]{2}{*}{ Location } & \multirow[t]{2}{*}{ Lesion Size } & \multirow[t]{2}{*}{ Follow-Up } & \multicolumn{2}{|c|}{ Clinical Results } & \multirow{2}{*}{$\begin{array}{c}\begin{array}{c}\text { Radiologic Results/ } \\
\text { Histological } \\
\text { Evaluation }\end{array} \\
\text { Postop }\end{array}$} & \multirow[t]{2}{*}{ Complications/Reoperation } \\
\hline & & & & & & Preop & Postop & & \\
\hline $\begin{array}{l}\text { Perdisa et al.2018, } \\
\text { Am J Sport Med } \\
\text { [31] }\end{array}$ & $\begin{array}{l}\text { Case Series, } \\
\text { Level IV }\end{array}$ & $\begin{array}{c}27 \text { patients; } \\
25.5 \pm 7.7 \text { years }\end{array}$ & $\begin{array}{l}27 \text { lesions: } 17 \mathrm{MFC} \text {, } \\
10 \mathrm{LFC}\end{array}$ & $3.4 \pm 2.2 \mathrm{~cm}^{2}$ & $\begin{array}{l}12,24,36,48 \\
\text { and } 60 \text { months } \\
\text { after surgery }\end{array}$ & $\begin{array}{l}\text { IKDC: } 48.4 \pm 17.8 \\
\text { Tegner: } 2.4 \pm 1.7 \\
5.7 \pm 2.2 \text { (before onset } \\
\text { of symptoms) }\end{array}$ & $\begin{array}{c}\text { IKDC: } \\
12 \text { months: } p<0.0005 \text { vs preop * } \\
24 \text { months: } p<0.0005 \text { vs preop } \\
36 \text { months: } p<0.00005 \text { vs preoop * } \\
48 \text { months: } p<0.0005 \text { vs preop * } \\
60 \text { months: } p<0.0005 \text { vs preop and } \\
\text { vs } 24 \text { months * } \\
\text { Tegner: } \\
12 \text { months: non-significant } \\
\text { improvement vs preop } \\
24 \text { months: } 4.4 \pm \pm 1.6(p=0.001)^{*} \\
60 \text { months: } 5.0 \pm 1.7(p<0.0005)^{*}\end{array}$ & $\begin{array}{c}\text { MOCART: } \\
24 \text { months: } 74.2 \pm 16.2 \\
60 \text { months: } 81.4 \pm 11.8\end{array}$ & None \\
\hline $\begin{array}{l}\text { Verdonk et al., } \\
\text { 2015, Bone Joint J } \\
\text { [32] }\end{array}$ & $\begin{array}{l}\text { Case Series, } \\
\text { Level IV }\end{array}$ & $\begin{array}{c}38 \text { patients; } \\
30.5 \pm 11.9 \\
\text { years }\end{array}$ & $\begin{array}{l}38 \text { lesions: } \\
23 \text { MFC, } \\
7 \text { LFC, } \\
5 \text { patella, } \\
3 \text { trochlea }\end{array}$ & $3.7 \pm 2.4 \mathrm{~cm}^{2}$ & $\begin{array}{c}3,6,12,18,24 \\
\text { months }\end{array}$ & $\begin{array}{c}\text { KOOS: } 213.9 \pm 88.3 \\
\text { Tegner: } 3.1 \pm 2.5\end{array}$ & $\begin{array}{c}\text { KOOS: } \\
3 \text { months: } 261.2 \pm 98.8 *(p=0.01) \\
6 \text { months: } 295.9 \pm 106.1 *(p=0.01) \\
12 \text { months: } 328.2 \pm 105.7 *(p=0.01) \\
18 \text { months: } 335.8 \pm 100.9 *(p=0.01) \\
24 \text { months: } 356.1 \pm 96.9 *(p=0.01) \\
\text { Tegner: } \\
\text { 3 months: } 1.9 \pm 1.9 *(p=0.01) \\
6 \text { months: } 2.5 \pm 1.9(p=0.09) \\
12 \text { months: } 3.4 \pm 1.9(p=0.46) \\
18 \text { months: } 3.4 \pm 1.8(p=0.27) \\
24 \text { months: } 3.8 \pm 1.9 *(p=0.03)\end{array}$ & $\begin{array}{l}\text { MOCART: Significant } \\
\text { improvement at } 3,12 \\
\text { and } 24 \text { months after } \\
\text { surgery. }\end{array}$ & $\begin{array}{c}2 \text { failures: total knee } \\
\text { arthroplasty at } 14 \text { and } 20 \\
\text { months } \\
3 \text { complications: } \\
-1 \text { further arthroscopy due to } \\
\text { hypertrophy } \\
-2 \text { joint stiffress } \\
\text { treated with knee mobilization } \\
\text { under anesthesia }\end{array}$ \\
\hline
\end{tabular}

* Statistical significant difference vs. pre-op; † statistical significant difference vs. previous follow-up; MFC: Medial femoral condyle; LFC: Lateral femoral condyle; IKDC: International Knee Documentation Committee; EQ-VAS: EuroQol-visual analogue scales; VAS: Visual analogue scales; KOOS: Knee Injury and Osteoarthritis Outcome Score; MOCART: Magnetic resonance observation of cartilage repair tissue; SPECT/CT: Single photon emission computed tomography/Computed tomography. 


\subsection{Demographic Results}

Data from a total number of 471 patients were included in this systematic review. The mean age was $34.07 \pm 5.28$ years. The treatment involved 500 lesions divided as follows: $202(40.4 \%)$ medial femoral condyles, $107(21.4 \%)$ lateral femoral condyles, 28 (5.6\%) tibial plateaus, 46 (9.2\%) trochleas, $74(14.8 \%)$ patellas, and $43(8.6 \%)$ unspecified femoral condyles. Mean superficial lesion size was $3.6 \pm 0.85 \mathrm{~cm}^{2}$. All lesions were classified as Grade III or IV (ICRS classification), except for two studies in which patients were treated for spontaneous osteonecrosis of the knee (SPONK) or unicompartmental knee osteoarthritis grade 3 (Kellgren-Lawrence classification). Only four studies [23,26,29,31] (25\%) reported a follow-up longer than 24 months and only two studies [23,27] (12.5\%) reported a post-operative histological evaluation. Almost all the articles reported clinical data using Tegner score (15/16-93.75\%) [15,17-30] or International Knee Documentation Committee score (IKDC) (14/16-87.5\%) [15-27,29]. Other reported clinical scores were Knee Injury and Osteoarthritis Outcome Score (KOOS), EuroQol-visual analog scales (EQ-VAS), Cincinnati, and Lysholm [17-19,30,32]. Tegner activity level scale is a graduated list of activities of daily living, recreation, and competitive sports. The patient is asked to select the level of participation that best describes their current level of activity and that before injury. The score varies from $0-10$. A score of 0 represents sick leave or disability pension, whereas a score of 10 corresponds to participation in national and international elite competitive sports. The International Knee Documentation Committee is a knee-specific patient-reported outcome measure. The IKDC Questionnaire is a subjective scale that provides patients with an overall function score. The questionnaire looks at 3 categories: symptoms, sports activity, and knee function. Scores are obtained by summing the individual items, then transforming the crude total to a scaled number that ranges from 0 to 100 . The Cincinnati knee-rating system has 11 components including sections that measure physical examination, instrumented knee stability, testing, and radiographic findings. Lysholm consists of 8 different items on a 100-point scale with 25 points each attributed to instability and pain. The EQ VAS records the patient's self-rated health on a vertical visual analog scale. This can be used as a quantitative measure of health outcome that reflects the patient's own judgment.

\subsection{Clinical Outcome in Early Postoperative Period (12 Months)}

Thirteen studies (81.25\%) [17,19-25,27,28,30-32] showed a clinical significant improvement (IKDC, Lysholm and KOOS) at 12 months follow-up in comparison with the pre-operative value $(p<0.05)$. Only one study $(6.25 \%)$ reported no clinical significant improvement [18] $(p>0.05)$. A significant improvement $(p<0.05)$ was also reported comparing data at 6 and 12 months follow up (measured by IKDC) in one study (6.25\%) [20]. Eleven studies (68.75\%) [19-22,24,25,27,28,30-32] collected Tegner score data at one year after surgery, of which only seven $(43.75 \%)[20-22,24,25,27,28]$ showed a significant improvement $(p<0.05)$, while four studies $(25 \%)[19,30-32]$ showed similar or inferior score at follow-up with respect to pre-operative value.

\subsection{Clinical Outcome at Intermediate Follow-Up (24 Months)}

Two years after surgery, thirteen (81.25\%) $[17,19-28,31,32]$ studies reported a clinical (IKDC, Lysholm, and KOOS) significant improvement compared with pre-operative values $(p<0.05)$. Likewise, early follow-up, the study of Brix et al. reported no significant clinical improvement [18]. Furthermore, the most interesting data are found in seven studies (43.75\%) $[17,22-25,27,28]$; a clinically significant improvement (IKDC) was observed between 12 and 24 months $(p<0.05)$. For what concerns sports activity, eleven studies $(68.75 \%)[17,20,22-28,31,32]$ reported a significant clinical improvement of Tegner Score compared with pre-operative values $(p<0.05)$; in two studies $(12.5 \%)$ that also increased significantly between 12 and 24 months $[25,28](p<0.05)$. 


\subsection{Clinical Outcome in Long Term Follow-Up (>24 Months)}

Only four studies (25\%) [23,26,29,31] reported clinical follow-up longer than 24 months. All of them showed a significant improvement in IKDC and Tegner scores versus pre-operative value $(p<0.05)$, with stable results with respect to the previous follow-up. Only one study $(6.25 \%)$ [31] reported a significant improvement in IKDC score at 60 months after surgery with respect to 24-month follow-up $(p<0.05)$.

\subsection{Radiological Evaluation}

In four studies [19,20,22,29] (25\%) radiological results were not reported. Eleven studies $(68.75 \%)[17,18,21,23-28,31,32]$ evaluated the treatment using magnetic resonance observation of cartilage repair tissue (MOCART) score. The MOCART score was designed to evaluate the treatment of chondral and osteochondral lesions in as subjective a way as possible. This scale evaluates the appearance of the repaired tissue, the covering of the lesion, the integration of the margins, the intensity of the signal, and the state of the subchondral lamina. The score ranges from a minimum of 0 (worst possible result) to a maximum of 100 (best possible result).

In one study (6.25\%) [30], the authors performed an evaluation with single photon emission computed tomography/computed tomography (SPECT/CT).

Moreover, in one study (6.25\%) [18] T2 mapping was used to evaluate the cartilage quality. In three studies $(18.75 \%)[26,28,32]$, a significant improvement among consecutive follow-ups in MOCART score was reported $(p<0.05)$. The SPECT/CT performed at 12 months [30], reported a complete filling of the defect in $14 \%$, a partial filling in $14 \%$, and only minor filling in $72 \%$ of patients, while at 18 months the T2 mapping showed a mean zonal T2 index in the repair tissue of 0.9874 which differed significantly compared to the healthy control cartilage [18] $(p<0.05)$.

\subsection{Histological Evaluation}

Only two studies $(12.5 \%)[23,27]$ reported a histological evaluation. In the first study, Berruto et al. showed in all the specimens no residual scaffold [23]. Both subchondral bone and the mineralization process appeared normal in all the specimens evaluated with Mallory trichrome staining; Kon et al. reported complete biomaterial reabsorption and a hyaline-like tissue with a strong proteoglycan content and presence of collagen type II at 2-year follow-up [27].

\subsection{Minor Complications}

A total of 52 (11.1\%) minor complications were reported, of which 16 experienced joint stiffness, 5 fever, and 31 swelling and bleeding of the knee.

\subsection{Major Complications}

A total of 7 (1.48\%) major complications were reported of which 2 arthroscopic regularization/ shaving due to partial detachment of the scaffold, 1 arthroscopy due to a novel chondral lesion on the lateral condyle, and 1 arthroscopy to remove an infrapatellar ossicle, 1arthroscopy for graft loosening, and 2 graft hypertrophy.

\subsection{Failures}

A total of $16(3.39 \%)$ failures were reported.

\section{Discussion}

This systematic review aimed to evaluate existing literature concerning the use of MaioRegen for the treatment of osteochondral knee defects in humans, analyzing all relevant medical databases. The main finding of this systematic review is that MaioRegen can be considered a safe alternative treatment for an osteochondral defect with a low rate of complications or failures. 
MaioRegen is a tri-phasic scaffold incorporating a biomimetic design that attempts to resemble the structure of osteochondral tissue as closely as possible [11,13]. Using the nucleation of HA nanocrystals onto self-assembled collagen fibers, this biomaterial was generated so that it could mimic the hierarchical layered structure of osteochondral tissue while also resembling the composition of the extracellular matrix's (ECM) of cartilage and bone tissues [11,13].

The positive clinical results reported in this review highlight the advantages of having a hierarchical graded structure that mimics more closely the natural structure of the osteochondral tissue [10,11].

Histological results emphasize that no residual scaffold was identified with a complete resorption of the graft suggesting that the scaffold was progressively replaced and substituted by new tissue.

The studies included in this review demonstrated that MaioRegen is effective and reliable over time; in fact, almost all the studies reported a significant improvement in the first year after surgery, and seven studies $(43.75 \%)$ [17,22-25,27,28] reported further improvement between 12 and 24 months. Only one study $(6.25 \%)$ also reported a significant clinical improvement at 60 months of follow-up if compared with clinical results at 24 months [31] showing the durability of the outcomes.

A recent systematic review highlighted an increasing number of articles dealing with cell-free scaffolds both in clinical and preclinical studies [33]. This is not surprising because in recent years these scaffolds have gained more and more popularity and huge steps forward were made regarding biomaterials research with the development of a new generation of materials able to mimic the characteristics of human tissues and exploit the intrinsic tissue regeneration ability and avoid the risk linked to bacterial contamination and phenotype loss during extensive cell manipulation; therefore, reducing costs and simplifying the procedure. In this way, scaffolds that aim to restore osteochondral lesions were developed, with the challenge to guide the regeneration of 2 different tissues characterized by different healing potentials, and promising results have been shown in the clinical setting as well for the treatment of complex cases. This technique is robust and easy for surgeons to handle, and was reported to improve the healing of cartilage defects significantly [33].

On the other hand, there are still several problems associated with the safety and long-term effectiveness of these materials. Synthetic polymers can have potential problems of retention and degradation in situ. Biological materials potentially carry the risk of transmitting infectious agents and starting immunological reactions. Currently, in the literature, there are no studies comparing scaffold-based and scaffold-free approaches for the treatment of osteochondral defects and is still unknown which could be considered the gold standard. A scaffold-free cell delivery system can be considered an excellent alternative due to its simplicity both for development and implantation [33,34].

To date, in the literature, clinical results are available only regarding the use of other two synthetic osteochondral scaffolds: TruFit (Smith \& Nephew, Andover, MA, USA) and Agili-C (CartiHeal, 2009-Ltd, Kfar Sava, Israel) [35,36].

The TruFit plug is a synthetic, acellular scaffold and is predominantly made from a polylactide-coglycolide copolymer. The scaffold consists of two "phases." The bone phase contains calcium sulfate for stimulation of bone formation. Cartilage regeneration is instigated by the integration of cells and growth factors derived from the bone marrow that infiltrates the plug [37].

A systematic review published in 2015 reported clinical improvement at 12-months follow-up compared to pre-operatively using the TruFit plug; however, two studies reporting longer follow-up showed a deterioration of early improvement [38]. Radiological evaluation indicates favorable MRI findings regarding filling of the defect and incorporation with adjacent cartilage at 24 months follow-up using TruFit, but conflicting evidence exists on the properties of the newly formed overlying cartilage surface. None of the included studies showed evidence for bone ingrowth. The minimal histological data available confirmed these results [38].

Agili-C, an aragonite-base osteochondral scaffold, consists of two layers: (1) the bone phase represented by calcium carbonate in the aragonite crystalline form, and (2) the superficial cartilage phase composed by modified aragonite and biodegradable and biocompatible hyaluronic acid [36]. Preclinical analysis revealed the safety and potential of this scaffold, showing its biodegradability and 
intrinsic restorative potential. In particular, the scaffold was able to recruit cells from the surrounding tissue which allowed good regeneration of the entire osteochondral unit. In fact, it was demonstrated that chondro-progenitor cells with migratory ability are also present in pathological cartilage tissue. Their colony forming ability and their paracrine activity characterized by the release of chondrogenic, angiogenic and pro-mitogenic molecules [39] set the appropriate conditions to promote the scaffold integration. Therefore, the scaffold can be translated into the clinical setting as a technique for one-step implantation also without cell augmentation [36]. In a recent clinical study, 21 patients without severe osteoarthritis received tapered shaped aragonite-based scaffolds for the treatment of $2.5 \pm 1.7 \mathrm{~cm}^{2}$ knee cartilage defects. The control group consisted of 76 patients selected according to the same criteria from a database of patients who previously underwent implantation of cylindrical-shaped implants. A statistically significant improvement in all clinical scores was reported both in the tapered and cylindrical group. No difference could be detected between the improvement obtained with the two implant types, neither in the clinical nor in imaging evaluations. A difference could be detected, instead, in terms of the revision rate, which was lower in the tapered implant group with no implant removal $-0 \%$ vs. $8 / 76-10.5 \%$ failures in the cylindrical implants [40]. This can be reflective of a better preservation of the scaffold integrity during insertion, with the maintenance of a better press-fit and consequently an enhanced incorporation and in the end an improvement in terms of adverse events and failures while offering the same benefits of the more widely documented cylindrical implants in terms of clinical outcome [40].

Among the advantages of using synthetic scaffold is the lack of donor site morbidity. In fact, a systematic review analyzing the knee donor-site morbidity after mosaicplasty showed that the donor-site morbidity for knee-to-ankle (16.9\%) was greater than knee-to-knee (5.9\%) mosaicplasty procedures without any significant correlation between the rate of donor-site morbidity and size of the defect, and number and size of the plugs [41].

Another important finding in our review is the return to sport in patients who underwent MaioRegen treatment. After 24 months from surgery, eleven (68.75\%) [17,20,22-28,31,32] studies reported a clinical improvement in Tegner score, with further improvement in two studies (12.5\%) at 24 months $[25,28]$. Literature regarding return to sport using osteochondral scaffolds such as TruFit or Agili-C is scarce but a systematic review published in 2016 compared return-to-sport outcomes after microfracture (MFX), osteochondral autograft transfer, osteochondral allograft transplantation, and autologous chondrocyte implantation at a minimum follow-up of 2 years [42]. Data about 2549 athletes who had undergone cartilage restoration surgery showed that $76 \%$ returned to sport at mid-term follow-up. Osteochondral autograft transfer offered a faster recovery and appeared to have a higher rate of return to preinjury athletics but heterogeneity in lesion size, athlete age, and concomitant surgical procedures are important factors to consider when assessing individual athletes [42].

Our systematic review presents several limitations: first of all studies are level IV and only one is level III, highlighting the lack of randomized trials in this field and therefore limiting the quality and reliability of these findings.

Another limitation of our study is the fact that we have not considered the additional procedures that have been performed in patients; the purpose of the study was to analyze exclusively the use of the MaioRegen and considering the other surgical times would have required a stratification of the patients, creating possible bias, particularly in such a small cohorts of patients.

\section{Conclusions}

This systematic review describes the currently available evidence for the treatment of osteochondral knee defects with MaioRegen Osteochondral substitute reporting promising satisfactory and reliable results at mid-term follow-up while considering the return to sport. A low rate of complications and failure was reported, therefore, confirming the safety of this scaffold. Considering the low level of evidence of the study included in the review, this data does not support the superiority of the Maioregen in terms of clinical improvement at follow-up compared to conservative treatment or other 
cartilage techniques. Many doubts still exist regarding the ability to regenerate both hyaline cartilage formation and subchondral bone ingrowth, well-designed, large-scale, randomized controlled trials are needed to investigate the value of future synthetic scaffolds.

Author Contributions: All authors contributed equally to this paper with conception and design of the study, literature review and analysis, drafting and critical revision and editing, and final approval of the final version.

Funding: This research was funded by the Italian Ministry of Health "Ricerca Corrente".

Conflicts of Interest: The authors declare no conflict of interest.

\section{References}

1. Salzmann, G.M.; Niemeyer, P.; Hochrein, A.; Stoddart, M.J.; Angele, P. Articular Cartilage Repair of the Knee in Children and Adolescents. Orthop. J. Sports Med. 2018, 6. [CrossRef]

2. DiBartola, A.C.; Everhart, J.S.; Magnussen, R.A.; Carey, J.L.; Brophy, R.H.; Schmitt, L.C.; Flanigan, D.C. Correlation between histological outcome and surgical cartilage repair technique in the knee: A meta-analysis. Knee 2016, 23, 344-349. [CrossRef] [PubMed]

3. D'Ambrosi, R.; Ragone, V.; Ursino, N. What future in the treatment of osteochondral knee defects? Ann. Transl. Med. 2018, 6 (Suppl. 2), S100. [CrossRef]

4. Curl, W.W.; Krome, J.; Gordon, E.S.; Rushing, J.; Smith, B.P.; Poehling, G.G. Cartilage injuries: A review of 31,516 knee arthroscopies. Arthroscopy 1997, 13, 456-460. [CrossRef]

5. Widuchowski, W.; Widuchowski, J.; Trzaska, T. Articular cartilage defects: Study of 25,124 knee arthroscopies. Knee 2007, 14, 177-182. [CrossRef] [PubMed]

6. Brittberg, M.; Gomoll, A.H.; Canseco, J.A.; Far, J.; Lind, M.; Hui, J. Cartilage repair in the degenerative ageing Knee. Acta Orthop. 2016, 87, 26-38. [CrossRef] [PubMed]

7. D'Ambrosi, R.; Giacco, F.; Ragone, V.; Ursino, N. Arthroscopic treatment of osteochondral knee defects with resorbable biphasic synthetic scaffold: Clinical and radiological results and long-term survival analysis. Int. Orthop. 2018. [CrossRef] [PubMed]

8. de Girolamo, L.; Schönhuber, H.; Viganò, M.; Bait, C.; Quaglia, A.; Thiebat, G.; Volpi, P. Autologous Matrix-Induced Chondrogenesis (AMIC) and AMIC Enhanced by Autologous Concentrated Bone Marrow Aspirate (BMAC) Allow for Stable Clinical and Functional Improvements at up to 9 Years Follow-Up: Results from a Randomized Controlled Study. J. Clin. Med. 2019, 8, 392. [CrossRef]

9. Elmalı, N.; Tandoğan, R.; Demirel, M.; Bozkurt, M.; Beyzadeoglu, T. Cartilage repair strategies in the knee: A survey of Turkish surgeons. Acta Orthop. Traumatol. Turc. 2016, 50, 533-538. [CrossRef] [PubMed]

10. Bowland, P.; Ingham, E.; Jennings, L.; Fisher, J. Review of the biomechanics and biotribology of osteochondral grafts used for surgical interventions in the Knee. Proc. Inst. Mech. Eng. H 2015, 229, 879-888. [CrossRef]

11. Longley, R.; Ferreira, A.M.; Gentile, P. Recent Approaches to the Manufacturing of Biomimetic Multi-Phasic Scaffolds for Osteochondral Regeneration. Int. J. Mol. Sci. 2018, 19, 1755. [CrossRef] [PubMed]

12. de Girolamo, L.; Ragni, E.; Cucchiarini, M.; van Bergen, C.J.A.; Hunziker, E.B.; Chubinskaya, S. Cells, soluble factors and matrix harmonically play the concert of allograft integration. Knee Surg. Sports Traumatol. Arthrosc. 2018. [CrossRef]

13. Kon, E.; Delcogliano, M.; Filardo, G.; Altadonna, G.; Marcacci, M. Novel nano-composite multi-layered biomaterial for the treatment of multifocal degenerative cartilage lesions. Knee Surg. Sports Traumatol. Arthrosc. 2009, 17, 1312-1315. [CrossRef]

14. Moher, D.; Liberati, A.; Tetzlaff, J.; Altman, D.G.; PRISMA Group. Preferred reporting items for systematic reviews and meta-analyses: The PRISMA statement. PLoS Med. 2009, 6, e1000097. [CrossRef] [PubMed]

15. Marx, R.G.; Wilson, S.M.; Swiontkowski, M.F. Updating the assignment of levels of evidence. J. Bone Joint Surg. Am. 2015, 97, 1-2. [CrossRef] [PubMed]

16. Moher, D.; Shamseer, L.; Clarke, M.; Ghersi, D.; Liberati, A.; Petticrew, M.; Shekelle, P.; Stewart, L.A.; PRISMA-P Group. Preferred reporting items for systematic review and meta-analysis protocols (PRISMA-P) 2015 statement. Syst. Rev. 2015, 4, 1. [CrossRef]

17. Delcogliano, M.; de Caro, F.; Scaravella, E.; Ziveri, G.; De Biase, C.F.; Marotta, D.; Marenghi, P.; Delcogliano, A. Use of innovative biomimetic scaffold in the treatment for large osteochondral lesions of the Knee. Knee Surg. Sports Traumatol. Arthrosc. 2014, 22, 1260-1269. [CrossRef] [PubMed] 
18. Brix, M.; Kaipel, M.; Kellner, R.; Schreiner, M.; Apprich, S.; Boszotta, H.; Windhager, R.; Domayer, S.; Trattnig, S. Successful osteoconduction but limited cartilage tissue quality following osteochondral repair by a cell-free multilayered nano-composite scaffold at the Knee. Int. Orthop. 2016, 40, 625-632. [CrossRef]

19. Berruto, M.; Ferrua, P.; Uboldi, F.; Pasqualotto, S.; Ferrara, F.; Carimati, G.; Usellini, E.; Delcogliano, M. Can a biomimetic osteochondral scaffold be a reliable alternative to prosthetic surgery in treating late-stage SPONK? Knee 2016, 23, 936-941. [CrossRef]

20. Kon, E.; Filardo, G.; Venieri, G.; Perdisa, F.; Marcacci, M. Tibial plateau lesions. Surface reconstruction with a biomimetic osteochondral scaffold: Results at 2 years of follow-up. Injury 2014, 45, S121-S125. [CrossRef] [PubMed]

21. Di Martino, A.; Kon, E.; Perdisa, F.; Sessa, A.; Filardo, G.; Neri, M.P.; Bragonzoni, L.; Marcacci, M. Surgical treatment of early knee osteoarthritis with a cell-free osteochondral scaffold: Results at 24 months of follow-up. Injury 2015, 46, S33-S38. [CrossRef]

22. Filardo, G.; Kon, E.; Perdisa, F.; Di Matteo, B.; Di Martino, A.; Iacono, F.; Zaffagnini, S.; Balboni, F.; Vaccari, V.; Marcacci, M. Osteochondral scaffold reconstruction for complex knee lesions: A comparative evaluation. Knee 2013, 20, 570-576. [CrossRef] [PubMed]

23. Berruto, M.; Delcogliano, M.; de Caro, F.; Carimati, G.; Uboldi, F.; Ferrua, P.; Ziveri, G.; De Biase, C.F. Treatment of Large Knee Osteochondral Lesions with a Biomimetic Scaffold: Results of a Multicenter Study of 49 Patients at 2-Year Follow-up. Am. J. Sports Med. 2014, 42, 1607-1617. [CrossRef]

24. Perdisa, F.; Filardo, G.; Sessa, A.; Busacca, M.; Zaffagnini, S.; Marcacci, M.; Kon, E. One-Step Treatment for Patellar Cartilage Defects With a Cell-Free Osteochondral Scaffold: A Prospective Clinical and MRI Evaluation. Am. J. Sports Med. 2017, 45, 1581-1588. [CrossRef]

25. Filardo, G.; Kon, E.; Di Martino, A.; Busacca, M.; Altadonna, G.; Marcacci, M. Treatment of knee osteochondritis dissecans with a cell-free biomimetic osteochondral scaffold: Clinical and imaging evaluation at 2-year follow-up. Am. J. Sports Med. 2013, 41, 1786-1793. [CrossRef] [PubMed]

26. Kon, E.; Filardo, G.; Di Martino, A.; Busacca, M.; Moio, A.; Perdisa, F.; Marcacci, M. Clinical results and MRI evolution of a nano-composite multilayered biomaterial for osteochondral regeneration at 5 years. Am. J. Sports Med. 2014, 42, 158-165. [CrossRef] [PubMed]

27. Kon, E.; Delcogliano, M.; Filardo, G.; Busacca, M.; Di Martino, A.; Marcacci, M. Novel nano-composite multilayered biomaterial for osteochondral regeneration: A pilot clinical trial. Am. J. Sports Med. 2011, 39, 1180-1190. [CrossRef] [PubMed]

28. Kon, E.; Filardo, G.; Perdisa, F.; Di Martino, A.; Busacca, M.; Balboni, F.; Sessa, A.; Marcacci, M. A one-step treatment for chondral and osteochondral knee defects: Clinical results of a biomimetic scaffold implantation at 2 years of follow-up. J. Mater. Sci. Mater. Med. 2014, 25, 2437-2444. [CrossRef] [PubMed]

29. Marcacci, M.; Zaffagnini, S.; Kon, E.; Marcheggiani Muccioli, G.M.; Di Martino, A.; Di Matteo, B.; Bonanzinga, T.; Iacono, F.; Filardo, G. Unicompartmental osteoarthritis: An integrated biomechanical and biological approach as alternative to metal resurfacing. Knee Surg. Sports Traumatol. Arthrosc. 2013, 21, 2509-2517. [CrossRef] [PubMed]

30. Mathis, D.T.; Kaelin, R.; Rasch, H.; Arnold, M.P.; Hirschmann, M.T. Good clinical results but moderate osseointegration and defect filling of a cell-free multi-layered nano-composite scaffold for treatment of osteochondral lesions of the Knee. Knee Surg. Sports Traumatol. Arthrosc. 2018, 26, 1273-1280. [CrossRef]

31. Perdisa, F.; Kon, E.; Sessa, A.; Andriolo, L.; Busacca, M.; Marcacci, M.; Filardo, G. Treatment of Knee Osteochondritis Dissecans with a Cell-Free Biomimetic Osteochondral Scaffold: Clinical and Imaging Findings at Midterm Follow-up. Am. J. Sports Med. 2018, 46, 314-321. [CrossRef] [PubMed]

32. Verdonk, P.; Dhollander, A.; Almqvist, K.F.; Verdonk, R.; Victor, J. Treatment of osteochondral lesions in the knee using a cell-free scaffold. Bone Joint J. 2015, 97, 318-323. [CrossRef] [PubMed]

33. Kon, E.; Roffi, A.; Filardo, G.; Tesei, G.; Marcacci, M. Scaffold-based cartilage treatments: With or without cells? A systematic review of preclinical and clinical evidence. Arthroscopy 2015, 31, 767-775. [CrossRef] [PubMed]

34. Kon, E.; Filardo, G.; Roffi, A.; Andriolo, L.; Marcacci, M. New trends for knee cartilage regeneration: From cell-free scaffolds to mesenchymal stem cells. Curr. Rev. Musculoskelet. Med. 2012, 5, 236-243. [CrossRef] [PubMed]

35. Melton, J.T.; Wilson, A.J.; Chapman-Sheath, P.; Cossey, A.J. TruFit CB bone plug: Chondral repair, scaffold design, surgical technique and early experiences. Expert Rev. Med. Devices 2010, 7, 333-341. [CrossRef] 
36. Kon, E.; Filardo, G.; Robinson, D.; Eisman, J.A.; Levy, A.; Zaslav, K.; Shani, J.; Altschuler, N. Osteochondral regeneration using a novel aragonite-hyaluronate bi-phasic scaffold in a goat model. Knee Surg. Sports Traumatol. Arthrosc. 2014, 22, 1452-1464. [CrossRef]

37. Williams, R.J.; Gamradt, S.C. Articular cartilage repair using a resorbable matrix scaffold. Instr. Course Lect. 2008, 57, 563-571.

38. Verhaegen, J.; Clockaerts, S.; Van Osch, G.J.; Somville, J.; Verdonk, P.; Mertens, P. TruFit Plug for Repair of Osteochondral Defects-Where Is the Evidence? Systematic Review of Literature. Cartilage 2015, 6, 12-19. [CrossRef]

39. De Luca, P.; Kouroupis, D.; Viganò, M.; Perucca-Orfei, C.; Kaplan, L.; Zagra, L.; de Girolamo, L.; Correa, D.; Colombini, A. Human Diseased Articular Cartilage Contains a Mesenchymal Stem Cell-Like Population of Chondroprogenitors with Strong Immunomodulatory Responses. J. Clin. Med. 2019, 8, 423. [CrossRef]

40. Kon, E.; Robinson, D.; Verdonk, P.; Drobnic, M.; Patrascu, J.M.; Dulic, O.; Gavrilovic, G.; Filardo, G. A novel aragonite-based scaffold for osteochondral regeneration: Early experience on human implants and technical developments. Injury 2016, 47, S27-S32. [CrossRef]

41. Andrade, R.; Vasta, S.; Pereira, R.; Pereira, H.; Papalia, R.; Karahan, M.; Oliveira, J.M.; Reis, R.L.; Espregueira-Mendes, J. Knee donor-site morbidity after mosaicplasty-A systematic review. J. Exp. Orthop. 2016, 3, 31. [CrossRef] [PubMed]

42. Krych, A.J.; Pareek, A.; King, A.H.; Johnson, N.R.; Stuart, M.J.; Williams, R.J., 3rd. Return to sport after the surgical management of articular cartilage lesions in the knee: A meta-analysis. Knee Surg. Sports Traumatol. Arthrosc. 2017, 25, 3186-3196. [CrossRef] [PubMed]

(C) 2019 by the authors. Licensee MDPI, Basel, Switzerland. This article is an open access article distributed under the terms and conditions of the Creative Commons Attribution (CC BY) license (http://creativecommons.org/licenses/by/4.0/). 